\title{
DISTRIBUIÇÃO ESPACIAL E TEMPORAL DE UMA COMUNIDADE DE ANFÍBIOS ANUROS DO MUNICÍPIO DE RIO CLARO, SÃO PAULO, BRASIL
}

\section{TEMPORAL AND SPATIAL DISTRIBUTION IN AN ANURAN COMMUNITY IN MUNICIPALITY OF RIO CLARO, SP, BRAZIL}

\section{Toledo, L.F. ${ }^{1}$, Zina, J. ${ }^{1}$; Haddad, C.F.B. ${ }^{1}$}

${ }^{1}$ Departamento de Zoologia, Instituto de Biociências, Universidade Estadual Paulista, C. P. 199, CEP 13506-970, Rio Claro, São Paulo, Brasil. (toledolf@hotmail.com).

\section{RESUMO}

A compreensão da composição e inter-relações das comunidades faunísticas são fundamentais para a execução de medidas conservacionistas em ambientes florestais. No presente estudo nos avaliamos a comunidade de anfíbios de uma floresta estadual no município de Rio Claro, SP, com enfoque na biologia reprodutiva. Foram registradas 21 espécies, de quarto famílias: Bufonidae, Hylidae, Leptodactylidae e Microhylidae. As espécies da família Bufonidae reproduziram durante a estação fria e seca do ano e as demais somente durante a estação quente e chuvosa. Além de segregação temporal, algumas espécies dividiram o ambiente físico durante o período reprodutivo. Acentuada sobreposição temporal e espacial foi observada entre espécies filogeneticamente próximas, como entre Hyla nana e H. sanborni, entre Scinax fuscovarius e Scinax sp. (aff. similis), e entre Leptodactylus fuscus, L. mystacinus, e Leptodactylus sp. (gr. fuscus). Nestes casos, diferenças acústicas podem ter sido o principal fator responsável pela segregação reprodutiva. Ausência de segregação reprodutiva foi observada entre espécies do gênero $B u f o$, as quais foram vistas em amplexo interespecífico, apesar das diferenças acústicas entre seus cantos de anúncio.

PALAVRAS CHAVE. Historia natural, segregação temporal, espacial e acústica, Anura. 
The comprehension of structure and functioning of communities are fundamental for the execution of conservation efforts in forested areas. In the present study we evaluated a community of anurans in a State Forest in Southeast Brazil, emphasizing the reproductive biology of the species. It has been found 21 species, of four families: Bufonidae, Hylidae, Leptodactylidae, and Microhylidae. The species of the family Bufonidae reproduced during the dry and cold season, while other species reproduced only during the wet and warm season of the year. Besides temporal segregation, some species divided the physical environment during reproductive season. Strong temporal and spatial overlap was observed among phylogenetically close species (of the same genus), such as Hyla nana and H. sanborni, Scinax fuscovarius and Scinax sp. (aff. similis), and Leptodactylus fuscus, L. mystacinus, and Leptodactylus sp. (gr. fuscus). In these cases, acoustic differences may be the main factor responsible for the reproductive segregation. Lack of reproductive segregation was observed among species of the genus Bufo, which were found in interespecific amplexus, even with acoustic differences in their advertisement calls.

KEYWORDS. Natural history, temporal, seasonal and acoustic segregation, Anura.

\section{INTRODUÇÃO}

Nos últimos anos, alguns estudos sobre comunidades de anfíbios anuros têm sido realizados no Brasil (e.g. CARDOSO et al., 1989; CARDOSO \& VIELLARD, 1990; ARZABE et al., 1998; BERNARDE et al., 1999; ETEROVICK \& SAZIMA, 2000; BERNARDE \& MACHADO, 2001), especialmente no Estado de São Paulo (ver referências em HADDAD, 1998; e mais recentemente ver BERNARDE \& KOKUBUM, 1999; ROSSA-FERES \& JIM, 2001; BERTOLUCI \& RODRIGUES, 2002). Estudos como estes têm demonstrado vários níveis de segregação reprodutiva entre as espécies de uma mesma comunidade. As espécies podem apresentar desde partilha espacial e/ou temporal completa, até sobreposição total destes fatores (e.g. BERNARDE \& KOKUBUM, 1999; ROSSA-FERES \& JIM, 2001), podendo inclusive culminar em hibridação natural (ver HADDAD et al., 1988; 1990).

A partilha espacial inclui a exploração de uma grande diversidade de microambientes como sítios reprodutivos. Esses são utilizados de maneira diferente pelas espécies, sendo atualmente reconhecidas mais de 30 possibilidades para sítios de oviposição e desenvolvimento larvário (ver revisões em DUELLMAN \& TRUEB, 1994; STEBBINS \& COHEN, 1997). Já a partilha temporal, pode estar diretamente relacionada com as condições físicas da região. Para certas espécies a temporada reprodutiva está diretamente correlacionada com a temperatura ambiente e a disponibilidade de ambientes aquáticos temporários (e.g. BERNARDE \& ANJOS, 1999). Assim, as espécies 
adaptaram-se a microambientes sob condições específicas.

A constante degradação que os ecossistemas naturais vêm sofrendo, especialmente em virtude de ações antrópicas, implica na alteração ou eliminação completa dos microhabitats específicos explorados pelos anuros, sendo considerada o principal fator responsável pelos declínios populacionais observados em diversas espécies de anfíbios em escala global (revisões em BEEBEE, 1996; YOUNG et al., 2000).

Por outro lado, questiona-se se este declínio é um fenômeno recente, devido a mudanças climáticas e/ou perturbações antrópicas, ou trata-se de flutuações populacionais cíclicas naturais (POUGH et al., 1998). Este mesmo fenômeno foi observado em outros grupos animais como peixes e aves (STEBBINS \& COHEN, 1997). Desta maneira, estudos em longo prazo envolvendo comunidades são de fundamental importância para uma melhor compreensão e avaliação dessas flutuações populacionais.

Assim, o presente estudo visou caracterizar a comunidade de anfíbios anuros de uma Floresta semidecídua do interior do Estado de São Paulo, analisando a riqueza de espécies e a segregação reprodutiva temporal, espacial e acústica entre as espécies da comunidade, representando um primeiro conjunto de dados que poderão auxiliar no manejo e conservação das espécies de anuros registradas.

\section{MATERIAL E MÉTODOS}

A Floresta Estadual "Edmundo Navarro de Andrade" (FEENA), tombada em 1977, localiza-se no Município de Rio Claro, no planalto meridional do Estado de São Paulo (22⒉ $5^{\prime} \mathrm{S}, 47^{\circ} 33^{\prime} \mathrm{W}$; ca. $650 \mathrm{~m}$ ) e caracteriza-se por um verão quente e úmido e um inverno frio e seco. Conta com uma área de 2.222,80 ha e trata-se de um local de plantio de árvores exóticas, principalmente do gênero Eucalyptus (cerca de 180 espécies), dividido em talhões. Contudo, também possui remanescentes de matas ciliares e um crescente sub-bosque de flora nativa. O conjunto de águas da FEENA é composto por pequenos cursos d'água pertencentes à bacia hidrográfica do rio Corumbataí, sendo o principal deles o Ribeirão Claro, cortando-a no sentido norte-sul. Seus afluentes atingemno no sentido oeste-leste, como é o caso dos córregos Santo Antônio e Ibitinga, sendo que este último foi represado e hoje forma um grande açude, chamado de Lago Central (MACHADO, 1978).

Os principais sítios de reprodução visitados foram: (1) Campos abertos, gramados e alagados por chuvas. (2) Margens do Córrego Ibitinga, o qual apresenta no máximo $30 \mathrm{~cm}$ de profundidade e $5 \mathrm{~m}$ de largura na área estudada. Suas águas são cristalinas e correm com baixa velocidade. A vegetação ciliar apresenta-se preservada, contendo arbustos e árvores nativas. (3) Margens do Lago Central, um grande açude, de 
aproximadamente $40 \mathrm{~m}$ de diâmetro. A vegetação das margens do lago é composta, principalmente, por taboas (Typha sp.), ciperáceas (Fimbristylis autumnalis) e gramíneas (Hymenachne amplexicaulis). Sobre o lago predominam ninfeáceas (Nymphea sp.) e pinheirinhos d'água (Myriophyllum sp.). (4) Margens da Lagoa do Prona; esta lagoa apresenta cerca de $10 \mathrm{~m}$ de diâmetro com as margens em barranco e vegetação ciliar secundária. (5) Margens do Ribeirão Claro; este ribeirão apresenta cerca de $10 \mathrm{~m} \mathrm{de}$ largura e grande profundidade; suas águas são turvas e poluídas e a vegetação ciliar é composta principalmente por gramíneas e pequenos arbustos. (6) Pequenas poças temporárias de curta duração, localizadas às margens de córregos (fig. 1).

As coletas de dados foram realizadas semanalmente entre julho de 2001 e novembro de 2002, seguindo a metodologia de inventário completo de espécies (HEYER et al., 1994), tendo sido realizado um total de 52 visitas ao campo. A cada visita foram percorridos cerca de $12 \mathrm{~km}$ de automóvel por seis estradas da Floresta (fig. 1), perfazendo um total de $624 \mathrm{~km}$ ao longo do estudo. Ademais, foram realizadas caminhadas pelos sítios de vocalização totalizando cerca de 170 horas de observação, baseada em procura visual e auditiva.

As observações naturalísticas tiveram início pouco antes do ocaso e término entre 23:00 e 24:00 horas. Nas observações noturnas foram utilizadas lanternas de luz branca. As vocalizações das espécies foram gravadas com um gravador cassete, Panasonic ${ }^{\circledR}$ (modelo RQ-L30), utilizando-se um microfone cardióide externo Coby ${ }^{\circledR}$ (modelo CM-P24), para auxiliar na identificação das espécies em laboratório.

As coletas foram restritas ao material testemunho (de gravações ou entidades consideradas como ainda não descritas), sendo os animais transportados em sacos plásticos umedecidos. Foram fixados em laboratório e depositados na coleção de anuros Célio F. B. Haddad (CFBH), Departamento de Zoologia, I.B., Universidade Estadual Paulista (UNESP), Rio Claro, São Paulo, Brasil. A caracterização dos sítios de vocalização (altura, tipo de substrato utilizado e distância em relação à água) foi registrada a cada encontro com os indivíduos.

Dados sobre pluviosidade (total mensal), temperatura e umidade relativa (médias mensais) foram fornecidos pelo CEAPLA (Centro de Análise e Planejamento Ambiental), situado a aproximadamente $2 \mathrm{~km}$ da área de estudo (ver fig. 1). Foi realizada uma análise de regressão entre os dados ambientais (temperatura, umidade relativa e pluviosidade) e o número de espécies em atividade de vocalização sendo considerados significativos os valores de $P<0,05$ (ZAR, 1999). A curva do coletor foi calculada a partir da média de 50 aleatorizações geradas com os dados obtidos em campo pelo programa EstimateS versão $6.0 \mathrm{~b} 1 \mathrm{e}$ a riqueza absoluta foi estimada por meio do índice Jackknife de primeira ordem (COLWELL, 2000). 


\section{RESULTADOS}

Foram encontradas 21 espécies de anfíbios anuros, das quais apenas duas (Physalaemus fuscomaculatus e $P$. nattereri) não foram encontradas em atividade de vocalização e duas foram consideradas como espécies ainda não descritas [Scinax sp. (aff. similis) e Leptodactylus sp. (gr. fuscus)]. As espécies encontradas pertencem a quatro famílias distintas, tendo sido encontrados dois Bufonidae, nove Hylidae, oito Leptodactylidae e dois Microhylidae (tab. 1).

Através da procura ativa a pé foram encontradas 90,48\% das espécies (19 espécies), das quais nove (Hyla biobeba, H. faber, H. nana, H. minuta, H. sanborni; Leptodactylus mystacinus, Physalaemus centralis, Chiasmocleis albopunctata e Elachistocleis cf. ovalis) só puderam ser encontradas por este método. A procura por automóvel resultou no encontro de 57,14 \% das espécies da área (12 espécies), sendo que duas (Physalaemus fuscomaculatus e $P$. nattereri) só foram encontradas por este método.

Foi realizada a curva do coletor, relacionando o número de espécies acumuladas com o número de visitas realizadas ao campo. As aleatorizações realizadas resultaram em uma curva que aponta um crescente número de espécies encontradas ao longo das visitas, praticamente atingindo um platô horizontal (fig. 2). Foi estimada a riqueza de $23 \pm 1,4$ espécies (amplitude $=22$ a 24 espécies) de anfíbios para a FEENA.

Ao longo dos 17 meses de estudo, o número de espécies em atividade de vocalização foi influenciado pela pluviosidade total mensal $\left(\mathrm{r}^{2}=0,322 ; P=0,0176\right)$ (fig. $3 \mathrm{~A})$ e pela temperatura média mensal $\left(\mathrm{r}^{2}=0,508 ; P=0,0013\right)$ (fig. $\left.3 \mathrm{~B}\right)$, mas não foi influenciado pela umidade relativa média mensal $(r=0,001 ; P=0,996)$. Nenhuma espécie se reproduziu continuamente ao longo do ano, demonstrando preferência, ou pela estação seca e fria (espécies do gênero Bufo), ou pela estação quente e úmida (demais espécies; ver tab. 1). Todas as espécies da FEENA apresentaram atividade de vocalização exclusivamente crepuscular e noturna.

Com relação à sazonalidade, as espécies puderam ser reunidas em três grupos, de acordo com seu padrão reprodutivo observado em campo (sensu WELLS, 1977b): (I) espécies com padrão reprodutivo prolongado, cuja atividade de vocalização se manteve por mais de cinco meses (Hyla albopunctata, H. nana, H. sanborni e Physalaemus cuvieri); (II) espécies que apresentaram padrão reprodutivo intermediário, com atividade de vocalização que durou entre poucas semanas até quatro meses [Bufo cf. crucifer, B. schneideri, Hyla biobeba, H. faber, H. minuta, Leptodactylus fuscus, L. labyrinthicus, L. mystacinus e Leptodactylus sp. (gr. fuscus)]; e (III) espécies que apresentaram padrão reprodutivo explosivo, permanecendo em atividade de vocalização por uma ou apenas algumas noites [Scinax fuscovarius, Scinax sp. (aff. similis), Osteocephalus langsdorffii, Physalaemus centralis, P. fuscomaculatus, P. nattereri, 
Chiasmocleis albopunctata e Elachistocleis cf. ovalis].

Quanto à distribuição espacial, as espécies divergiram não só na utilização de macroambientes para reprodução, como também na utilização de microambientes específicos utilizados como sítios de vocalização, identificados a seguir:

1) Campos abertos (CA1, CA2 e CA3 da fig. 1) com presença de pequenas poças temporárias. Estes foram os ambientes com maior riqueza de espécies, sendo que 14 espécies foram registradas vocalizando em três microambientes preferenciais:

1.1) Nível da água com o corpo parcialmente submerso ou flutuando. Foram registradas as espécies: Physalaemus centralis, Physalaemus cuvieri, Chiasmocleis albopunctata e Elachistocleis cf. ovalis. Embora sintópicos, os machos das duas espécies de Physalaemus vocalizaram em dias distintos. Entretanto, os microhilídeos ocorreram de maneira sincronotópicos.

1.2) Na vegetação arbustiva ou arbórea, marginal ou sobre a água, no estrato inferior, abaixo de 1,5 m de altura, foram registradas as seguintes espécies: Hyla nana, H. minuta, H. sanborni, eventualmente Scinax fuscovarius e Scinax sp. (aff. similis), todas ocorrendo de maneira sincronotópicas. As duas espécies de Scinax emitiram vocalizações territoriais muito semelhantes (não gravadas).

1.3) No estrato superior, acima de 1,5 m, foram registradas as espécies: Hyla faber, Osteocephalus langsdorffii e esporadicamente Scinax sp. (aff. similis). Essas vocalizaram em datas distintas.

1.4) Sobre o solo seco, às margens das poças foram registradas as seguintes espécies sincronotópicas: Scinax fuscovarius, Scinax sp. (aff. similis), Leptodactylus fuscus, Leptodactylus mystacinus e Leptodactylus sp. (gr. fuscus).

2) Margens do Córrego Ibitinga ou do Ribeirão Claro. Estes macroambientes foram os de menor riqueza dentro da FEENA, utilizados por apenas três espécies, que divergiram quanto aos microambientes ocupados:

2.1) No estrato arbóreo, acima de $4 \mathrm{~m}$ de altura, apenas Hyla biobeba foi registrada.

2.2) Margens de remansos sobre solo levemente encharcado, foram registrados Leptodactylus labyrinthicus e Leptodactylus sp. (gr. fuscus), as quais ocorreram em trechos distintos dos corpos d'água.

3) Margens do Lago Central (LC) e Lagoa do Prona (LP); estes macroambientes perenes foram explorados da seguinte maneira:

3.1) No nível da água parcialmente submersos, sustentados ou agarrados à vegetação 
aquática, ocorreram: Bufo cf. crucifer que ocorreu tanto no LC como na LP e Bufo schneideri e Physalaemus cuvieri, os quais ocorreram somente no LC. Em duas ocasiões foram observadas tentativas de amplexo de machos de Bufo cf. crucifer por machos de Bufo schneideri, o qual realizava procura ativa por fêmeas.

3.2) Sobre a vegetação marginal foram registrados os hilídeos: Hyla albopunctata, que ocorreu tanto no LC como na LP, e Hyla nana, H. minuta, H. sanborni, Scinax fuscovarius e Scinax sp. (aff. similis), os quais ocorreram apenas no LC, sendo que neste ambiente houve um curto período de sobreposição temporal entre todas as espécies (tab. $1)$.

Foi possível agrupar as espécies encontradas em quatro categorias conforme seus modos reprodutivos (sensu DUELLMAN \& TRUEB, 1994):

Modo 1: ovos e girinos desenvolvem-se em corpos de água lênticos. Foram registradas 11 espécies pertencentes a esta categoria (todas as espécies das Famílias Bufonidae, Hylidae e Microhylidae, exceto Hyla biobeba e H. faber).

Modo 3: ovos e os primeiros estágios larvais aquáticos se desenvolvem em cavidades construídas ou naturais, que são posteriormente alagadas. O desenvolvimento completase em corpos de água lênticos ou lóticos. Duas espécies, Hyla biobeba e H. faber, apresentaram este modo.

Modo 8: ovos depositados em ninhos de espuma aquáticos; os girinos desenvolvem-se em poças. Cinco espécies (as quatro espécies de Physalaemus e Leptodactylus labyrinthicus) enquadram-se neste modo.

Modo 21: ovos e primeiros estágios larvais desenvolvem-se em ninhos de espuma depositados em tocas subterrâneas construídas e completam seu desenvolvimento em poças ou riachos. Três espécies apresentaram este modo (espécies de Leptodactylus do grupo de L. fuscus).

\section{DISCUSSÃO}

Os métodos de levantamento de fauna utilizados (a pé e com auxílio de automóvel) revelaram-se complementares, já que algumas espécies só puderam ser registradas através de um ou de outro método. Entretanto, constatou-se que a procura ativa a pé foi mais eficiente, contemplando quase a totalidade das espécies da área.

Apesar disso, como mostra a curva do coletor (fig. 2) e o resultado do índice de riqueza, o número de visitas ao campo pode ter sido insuficiente para a realização de 
um inventário completo da anurofauna da FEENA, havendo probabilidade de encontro de até três espécies adicionais no local de estudo. Além das limitações dos métodos utilizados (HEYER et al., 1994), algumas espécies podem não ter sido encontradas em decorrência de seus padrões reprodutivos explosivos (WELLS, 1977a), como por exemplo Physalaemus fuscomaculatus e $P$. nattereri, espécies que podem ter vocalizado nos dias em que não foram realizadas atividades de campo. A nossa metodologia de levantamento não abrangeu todas as áreas e ambientes úmidos da floresta (ver fig. 1), excluindo então a possibilidade de encontro de espécies adaptadas a estes ambientes. Ademais, é possível que algumas espécies possam ser raras em um determinado ambiente em decorrência de falta de condições adequadas para reprodução, estando de passagem pela área.

Verificamos que a atividade reprodutiva da comunidade da FEENA foi influenciada tanto pela temperatura como pela pluviosidade, assim como observado em outras localidades (e.g. AICHINGER, 1987; BERTOLUCI, 1998; BERNARDE \& ANJOS, 1999; BERNARDE \& KOKUBUM, 1999). Ademais, o padrão reprodutivo de algumas espécies parece estar relacionado a fatores intrínsecos à área de estudo. Este é o caso de Bufo cf. crucifer, que foi classificado como espécie de padrão reprodutivo prolongado em uma localidade (e.g. HADDAD, 1991; HADDAD \& SAZIMA, 1992) e de padrão intermediário em outras (presente estudo; POMBAL Jr, 1997). Além disso, diferentemente do que foi observado em outra localidade (ver BOKERMANN \& SAZIMA, 1973), Hyla biobeba apresentou período reprodutivo incomum na FEENA, isto é, vocalizou somente durante os meses quentes e chuvosos do ano. As demais espécies apresentaram atividade reprodutiva (baseada em vocalização) semelhante ao reportado por outros autores (e.g. HADDAD, 1991; ROSSA-FERES \& JIM, 1994; POMBAL Jr, 1997; BERNARDE \& ANJOS, 1999). Todavia, não descartamos a possibilidade de que nestes diferentes estudos, diferentes entidades taxonômicas possam ter sido tratadas sob o mesmo nome.

As espécies foram divididas em diferentes grupos, tanto quanto à estação do ano em que reproduziram, quanto ao padrão reprodutivo apresentado (sensu WELLS, 1977b). Contudo, apresentaram uma pronunciada sobreposição temporal interespecífica. Em alguns casos, essa sobreposição foi possível em decorrência de partilha de sítios de vocalização utilizados, modos reprodutivos distintos, ou mesmo quanto aos diferentes macroambientes explorados. Assim, a segregação reprodutiva pré-zigótica foi observada para diversas espécies da FEENA.

Apesar disso, observamos sincronotopia entre as espécies de Leptodactylus do grupo de L. fuscus (sensu HEYER, 1978) sem identificarmos divisão temporal ou espacial, uma situação que parece ser comum em diversas localidades onde duas ou mais espécies do grupo coexistem (obs. pessoal). Da mesma maneira, não foi registrada distribuição diferenciada entre os Microhylidae, os Hylidae do gênero Scinax e entre Hyla nana e H. sanborni, sendo a divergência acústica o único fator de segregação 
reprodutiva pré-zigótica reconhecido (para diferenças acústicas entre Hyla nana e $H$. sanborni veja MARTINS \& JIM, 2003). Isto não exclui a possibilidade de haver microdistribuição diferenciada entre tais espécies. Ademais, Scinax sp. (aff. similis) e $S$. fuscovarius emitiram vocalizações territoriais muito semelhantes, o que poderia gerar territorialidade interespecífica (ver SHWARTZ \& WELLS, 1984). No entanto, nossa observação não é suficiente para suportar essa hipótese.

A simpatria entre Bufo crucifer e B. schneideri foi observada em outra localidade (ver BERNARDE \& ANJOS, 1999), porém, neste caso as espécies apresentam segregação reprodutiva temporal e espacial. Na FEENA, contudo, as espécies ocorrem de maneira sincronotópica, havendo inclusive tentativas de amplexo interespecífico, o que pode ser conseqüência de um padrão reprodutivo explosivo (WELLS, 1977b). Essa falta de seletividade pode ocasionar hibridação natural entre espécies sincronotópicas, o que tem sido observado para espécies do gênero Bufo (e.g. SULLIVAN, 1986; HADDAD et al., 1988; 1990). Apesar disso, a segregação reprodutiva pré-zigótica entre as espécies da comunidade da FEENA foi evidenciada de diversas maneiras, acima discutidas.

A anurofauna da FEENA possui várias espécies típicas de áreas antropizadas, tais como Hyla albopunctata, H. faber, Leptodactylus fuscus, Physalaemus cuvieri, Scinax fuscovarius e Scinax sp. (aff. similis) (LUTZ, 1973; FEIO et al., 1998; HADDAD, 1998), além de Osteocephalus langsdorffii, que pode ter sido introduzida recentemente no planalto paulista em decorrência de atividade humana. Essa espécie é típica de florestas litorâneas úmidas e seu encontro em áreas isoladas do planalto paulista pode resultar de introdução acidental, por exemplo, através de plantas ornamentais trazidas do litoral para o planalto e que são usadas como abrigo por estes animais, como é o caso de Bromeliaceae.

Apesar disso, a FEENA ainda abriga espécies que podem ser naturais das florestas semidecíduas e cerrados típicos da região. Além disso, o fato de que quatro das espécies (ou 19\% das espécies da comunidade) não puderam ser identificadas com segurança, sendo duas consideradas como entidades ainda não descritas, revela o estado ainda incipiente em que se encontram os estudos taxonômicos no Brasil. Assim, mesmo considerando o atual estado frágil de conservação de áreas como a FEENA e sua baixa riqueza de espécies, remanescentes como este podem ser considerados como áreas importantes para a conservação dos anfíbios do Estado de São Paulo.

\section{AGRADECIMENTOS}

Cynthia Prado, Jaime Bertoluci e Paulo Bernarde e dois revisores anônimos pela leitura de versões prévias e críticas apresentadas, Renata Uduluschdt pela identificação do material vegetal e a todos aqueles que eventualmente participaram dos trabalhos de 
campo, principalmente, Débora Campos e Fábio Britto. IBAMA (licenças no. 072/2001 e 028/02-RAN) e COTEC-IF (proc. no. 42.241/2001) pelas autorizações concedidas. João Bosco Monteiro pela permissão para realização do projeto na FEENA. Ao CNPq e à FAPESP pelos auxílios concedidos.

\section{REFERÊNCIAS}

AICHINGER, M. Annual activity patterns of anurans in a seasonal Neotropical environment. Oecologia, Berlim, v. 71, p. 583-592, 1987.

ARZABE, C.; CARVALHO, C. X. C.; COSTA, M. A. G. Anuran assemblage in Castro forest ponds (Sergipe State, Brazil): comparative structure and calling activity patterns. Herpetologiacal Journal, London, v. 8, p. 111-113, 1998.

BEEBEE, T. J. C. Ecology and conservation of amphibians. London: Chapman \& Hall, 1996. $214 \mathrm{p}$.

BERNARDE, P. S.; ANJOS, L. Distribuição espacial e temporal da anurofauna no Parque Estadual Mata dos Godoy, Londrina, Paraná, Brasil. Comumunicações do museu de ciências e tecnologia, Porto Alegre, v. 12, p. 127-140, 1999.

BERNARDE, P. S.; MACHADO, R. A. Riqueza de espécies, ambientes de reprodução e temporada de vocalização da anurofauna em Três Barras do Paraná, Brasil (Amphibia: Anura). Cuadernos de Herpetología, Tucúman, v. 14, n. 2, p. 93- 104, 2001.

BERNARDE, P. S.; KOKUBUM, M. N. C. Anurofauna do Município de Guararapes, Estado de São Paulo, Brasil (Amphibia: Anura). Acta Biológica Leopoldensia, São Leopoldo, v. 21, n. 1, p. 89-97, 1999.

BERNARDE, P. S.; KOKUBUM, M. N. C.; MACHADO, R. A.; ANJOS, L. Uso de habitats naturais e antrópicos pelos anuros em uma localidade no Estado de Rondônia, Brasil (Amphibia: Anura). Acta Amazônica, Manaus, v. 29, n. 4, p. 555$562,1999$.

BERTOLUCI, J. Annual patterns of breeding activity in Atlantic rainforest anurans. Journal of Herpetology, Cincinnati, v. 32, n. 4, p. 607-611, 1998.

BERTOLUCI, J.; RODRIGUES, M. T. Seasonal patterns of breeding activity of Atlantic Rainforest anurans at Boracéia, Southeastern Brazil. Amphibia-Reptilia, Leiden, v. 23, p. 161-167, 2002.

BOKERMANN, W. C. A.; SAZIMA, I. Anfíbios da Serra do Cipó, Minas Gerais, Brasil. 
1 - espécies novas de "Hyla" (Anura, Hylidae). Revista Brasileira de Biologia, Rio de Janeiro, v. 33, n. 3, p. 329-336, 1973.

CARDOSO, A. J.; ANDRADE, G. V.; HADDAD, C. F. B. Distribuição espacial em comunidades de anfíbios (Anura) no sudeste do Brasil. Revista Brasileira de Biologia, Rio de Janeiro, v. 49, n. 1, p. 241-249, 1989.

CARDOSO, A. J.; VIELLARD, J. Vocalizações de anfíbios anuros de um ambiente aberto, em Cruzeiro do Sul, Estado do Acre. Revista Brasileira de Biologia, Rio de Janeiro, v. 50, n. 1, p. 229-242, 1990.

COLWELL, R. K. EstimateS 6.0b1: statistical estimation of species richness and shared species from samples. 2000. Disponível em: $<$ http://viceroy.eeb.uconn.edu/estimates $>$, acesso em: 22 ago. 2003.

DUELLMAN, W. E.; TRUEB, L. Biology of amphibians. 2.ed. Baltimore and London: McGraw-Hill, 1994. 670 p.

ETEROVICK, P. C.; SAZIMA, I. Structure of an anuran community in a montane meadow in southeastern Brazil: effects of seasonality, habitat, and predation. Amphibia-Reptilia, Leiden, v. 21, p. 439-461, 2000.

FEIO, R. N.; BRAGA, U. M. L.; WIEDERHECKER, H.; SANTOS, P. S. Anfíbios do Parque Estadual do Rio Doce (Minas Gerais). Viçosa: Universidade Federal de Viçosa, Instituto Estadual de Florestas, 1998. 32 p.

HADDAD, C. F. B. Biodiversidade dos anfíbios do Estado de São Paulo. In: CASTRO, R. M. C. (Ed). Biodiversidade do Estado de São Paulo, Brasil: síntese do conhecimento ao final do século XX. São Paulo: Editora Fapesp, 1998. v. 6, p. 17-26.

HADDAD, C. F. B.; ANDRADE, G. V.; CARDOSO, A. J. Anfíbios anuros no Parque Estadual da Serra da Canastra, Estado de Minas Gerais. Brasil Florestal, Brasília, v. 64, p. 9-20, 1988.

HADDAD, C. F. B.; CARDOSO, A. J.; CASTANHO, L. M. Hibridação natural entre Bufo ictericus e Bufo crucifer (Amphibia: Anura). Revista Brasileira de Biologia, Rio de Janeiro, v. 50, n. 3, p. 739-744, 1990.

HADDAD C. F. B.; SAZIMA, I. Anfíbios anuros da Serra do Japi. In: MORELLATO, L. C. P. ed. História natural da Serra do Japi. Campinas: Editora da Unicamp / FAPESP, 1992. p. 188-211.

HEYER, W. R. Systematics of group "fuscus" of the frog genus Leptodactylus (Amphibia, Leptodactylidae). Natural History Museum of Los Angeles County 
Science Bulletin, Los Angeles, v. 29, p. 1-85, 1978.

HEYER, R. W.; DONNELY, M. A.; MCDIARMID, R. W.; HAYEK, L. A. C.; FOSTER, M. S. Measuring and Monitoring Biological Diversity: standard methods for amphibians. Washington and London: Smithsonian Institution Press, 1994. $364 \mathrm{p}$.

LUTZ, B. Brazilian species of Hyla. Austin: University of Texas Press, 1973. 260 p.

MACHADO, I. L. O Horto Florestal "Navarro de Andrade". Rio Claro Sesquicentenária. Rio Claro: Museu de História e Pedagogia "Amador Bueno da Veiga", 1978. $350 \mathrm{p}$.

MARTINS, I. A.; JIM, J. Bioacustic analysis of advertisement call in Hyla nana and Hyla sanborni (Anura, Hylidae) In Botucatu, São Paulo Brazil. Brazilian Journal of Biology, São Carlos, v. 63, n. 3, p. 507-516, 2003.

POMBAL Jr, J. P. Distribuição espacial e temporal de anuros (Amphibia) em uma poça permanente na Serra de Paranapiacaba, Sudeste do Brasil. Revista Brasileira de Biologia, Rio de Janeiro, v. 57, n. 4, p. 583-594, 1997.

POUGH, F. H.; ANDREWS, R. M.; CADLE, J. E.; SAVITZKY, A. H.; WELLS, K. D. Herpetology. New Jersey, Prentice Hall, 1998. 577 p.

ROSSA-FERES, D. C.; JIM, J. Distribuição sazonal em comunidades de anfíbios anuros na região neotropical de Botucatu, São Paulo. Revista brasileira de Biologia, Rio de Janeiro, v. 54, n. 2, p. 323-334, 1994.

. Similaridade do sítio de vocalização em uma comunidade de anfíbios anuros na região noroeste do Estado de São Paulo, Brasil. Revista Brasileira de Zoologia, Rio de Janeiro, v. 18, n. 2, p. 439-454, 2001.

SHWARTZ, J. J.; WELLS, K. D. Interespecific acoustic interactions of the Neotropical treefrog Hyla ebraccata. Behavioral Ecology and Sociobiology, Boston, v. 14, p. 211-224, 1984.

STEBBINS, R. C.; COHEN, N. W. A Natural History of Amphibians. New Jersey: Princeton University Press, 1997. 316 p.

SULLIVAN, B. K. Hybridization between the toads Bufo microscaphus and Bufo woodhousii in Arizona: morphological variation. Journal of Herpetology, Cincinnati, v. 20, p. 11-21, 1986.

WELLS, K. D. The social behavior of anuran amphibians. Animal Behaviour, London, v. 25, p. 666-693, 1977a. 
WELLS, K. D. The courtship of frogs. In: TAYLOR D. H.; GUTTMAN S. I. (Eds.). The reproductive biology of amphibians. New York: Plenum, 1977b. 475 p.

YOUNG, B. E.; LIPS, K. R.; REASER, J. K.; IBÁÑES, R.; SALAS, A. W.; CEDEÑO, J. R.; COLOMA, L. A.; RON, S.; MARCA, E.; MEYER, J. R.; MUÑOZ, A.; BOLAÑOS, F.; CHAVES, G.; ROMO, D. Population declines and priorities for amphibian conservation in Latin America. Conservation Biology, Seatle, v. 15, n. 5, p. 1213-1223, 2000.

ZAR, J. H. Biostatistical Analysis. 4. ed. New Jersey: Prentice Hall, 1999. 663 p.

Tabela 1. Sazonalidade reprodutiva anual, baseada em vocalizações, dos machos anuros da Floresta Estadual "Edmundo Navarro de Andrade", Rio Claro, São Paulo, ao longo dos meses de estudo (de julho de 2001 a novembro de 2002). Os meses de julho, agosto, setembro e novembro representam os resultados acumulados de dois anos de estudo (2001 e 2002).

\begin{tabular}{|c|c|c|c|c|c|c|c|c|c|c|c|c|}
\hline \multirow{2}{*}{$\begin{array}{c}\text { Espécies } \\
\text { encontradas }\end{array}$} & \multicolumn{12}{|c|}{ Meses } \\
\hline & $\mathbf{J}$ & A & $\mathbf{S}$ & $\mathbf{O}$ & $\mathbf{N}$ & D & $\mathbf{J}$ & $\mathbf{F}$ & $\mathbf{M}$ & $\mathbf{A}$ & $\mathbf{M}$. & $\mathbf{J}$ \\
\hline \multicolumn{13}{|l|}{ Bufonidae } \\
\hline Bufo cf. crucifer & - & - & & & & & & & & & & \\
\hline Bufo schneideri & - & - & - & & & & & & & & & \\
\hline \multicolumn{13}{|l|}{ Hylidae } \\
\hline $\begin{array}{l}\text { Hyla } \\
\text { albopunctata }\end{array}$ & & & - & - & - & - & - & - & - & - & & \\
\hline Hyla biobeba & & & & - & - & & • & - & & & & \\
\hline Hyla faber & & & & & & & & & - & & & \\
\hline Hyla nana & & & - & - & - & - & - & - & - & & & \\
\hline Hyla minuta & & & & & - & & & & & & & \\
\hline Hyla sanborni & & & - & - & - & - & - & & - & & & \\
\hline $\begin{array}{l}\text { Osteocephalus } \\
\text { langsdorffii }\end{array}$ & & & & - & - & & & & & & & \\
\hline Scinax & & • & - & & - & & & & & & & \\
\hline
\end{tabular}




\begin{tabular}{|c|c|c|c|c|c|c|c|c|c|c|}
\hline fuscovarius & & & & & & & & & & \\
\hline $\begin{array}{l}\text { Scinax sp. (aff. } \\
\text { similis) }\end{array}$ & & & & - & & & & & & \\
\hline Leptodactylidae & & & & & & & & & & \\
\hline $\begin{array}{l}\text { Leptodactylus } \\
\text { fuscus }\end{array}$ & & & - & $\cdot$ & - & - & & & & \\
\hline $\begin{array}{l}\text { Leptodactylus } \\
\text { labyrinthicus }\end{array}$ & & & & - & & & & & & \\
\hline $\begin{array}{l}\text { Leptodactylus } \\
\text { mystacinus }\end{array}$ & & & - & $\cdot$ & - & & & & & \\
\hline $\begin{array}{l}\text { Leptodactylus } \\
\text { sp. (gr. fuscus) }\end{array}$ & & & - & . & - & & & & & \\
\hline $\begin{array}{l}\text { Physalaemus } \\
\text { centralis }\end{array}$ & & & & - & & & & & & \\
\hline $\begin{array}{l}\text { Physalaemus } \\
\text { cuvieri }\end{array}$ & & - & - & $\cdot$ & • & $\cdot$ & • & & & \\
\hline $\begin{array}{l}\text { Physalaemus } \\
\text { fuscomaculatus }\end{array}$ & & & & & & & & & & \\
\hline $\begin{array}{l}\text { Physalaemus } \\
\text { nattereri }\end{array}$ & & & & & & & & & & \\
\hline Microhylidae & & & & & & & & & & \\
\hline $\begin{array}{l}\text { Chiasmocleis } \\
\text { albopunctata }\end{array}$ & & & & - & & & & & & \\
\hline $\begin{array}{l}\text { Elachistocleis cf. } \\
\text { ovalis }\end{array}$ & & & & - & & - & & & & \\
\hline Estação do ano & $\begin{array}{c}\text { fria } \\
\mathrm{e} \\
\text { seca }\end{array}$ & & quer & nte $\mathrm{c}$ & $\mathrm{ecl}$ & uv & osa & & $\begin{array}{r}\text { fria } \\
\text { sec }\end{array}$ & \\
\hline $\begin{array}{l}\text { Número total de } \\
\text { espécies }\end{array}$ & 2 & 3 & $6 \quad 9$ & 16 & 7 & 7 & 4 & 4 & 10 & 00 \\
\hline
\end{tabular}




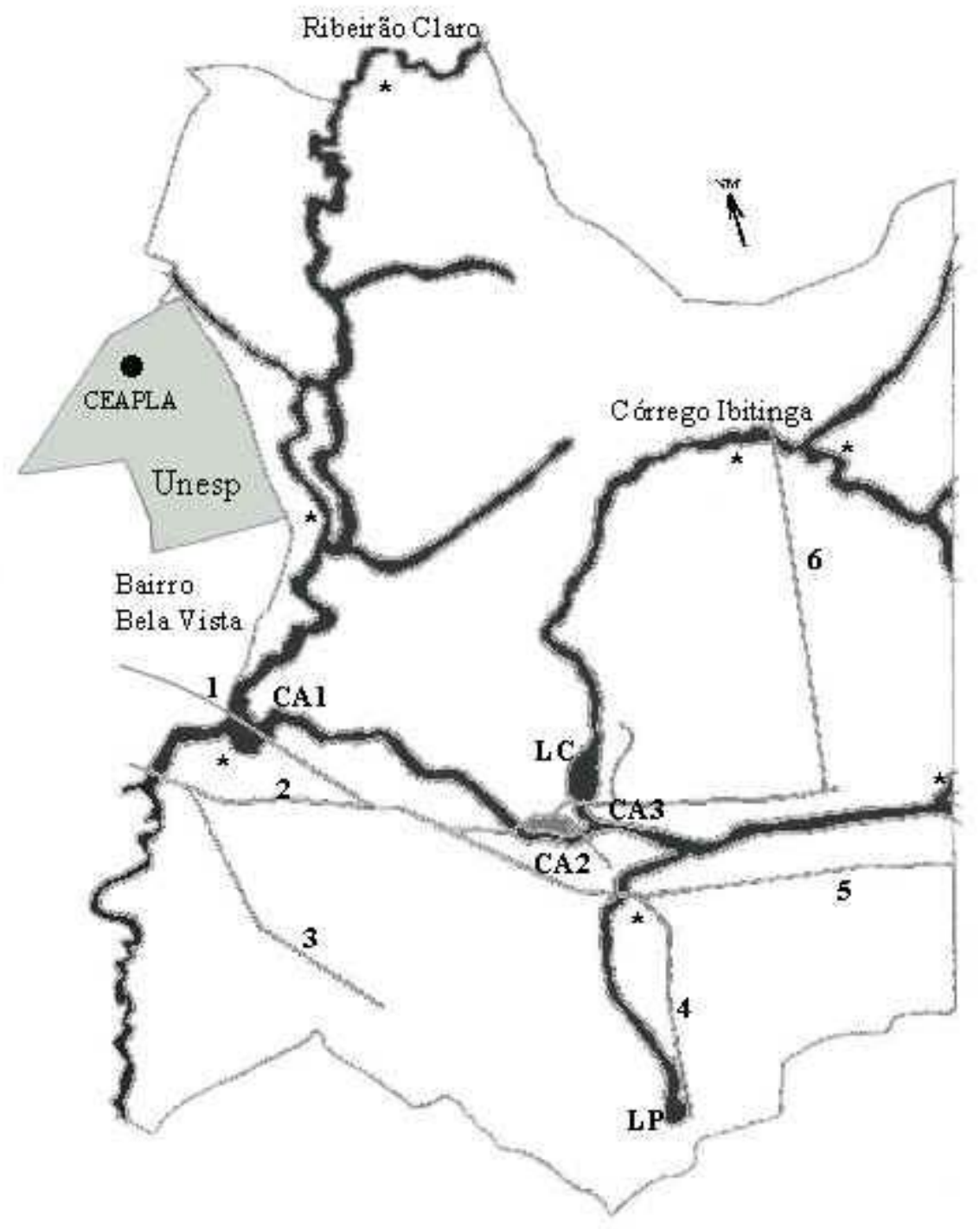

Figura 1. Mapa da Floresta Estadual "Edmundo Navarro de Andrade", Rio Claro, São Paulo, sendo: "CA1, CA2 e CA3" Campos abertos alagáveis; "LC" Lago Central; "LP" Lagoa do Prona; "1" a "6" estradas percorridas de carro; e "*" pequenas poças temporárias localizadas às margens dos córregos. 


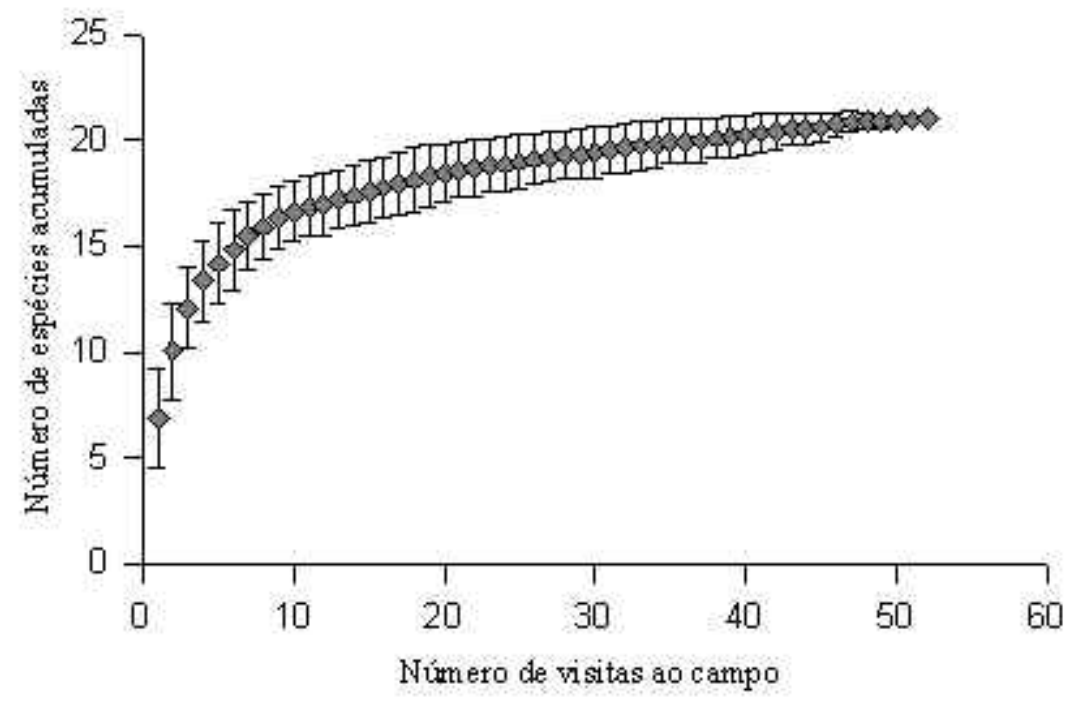

Figura 2. Curva do coletor, calculada com os dados obtidos entre julho de 2001 e novembro de 2002. Cada ponto expressa a média de 50 pontos gerados pelo simulador EstimateS versão $6.0 \mathrm{~b} 1$ e as barras indicam o desvio padrão calculado para cada ponto.
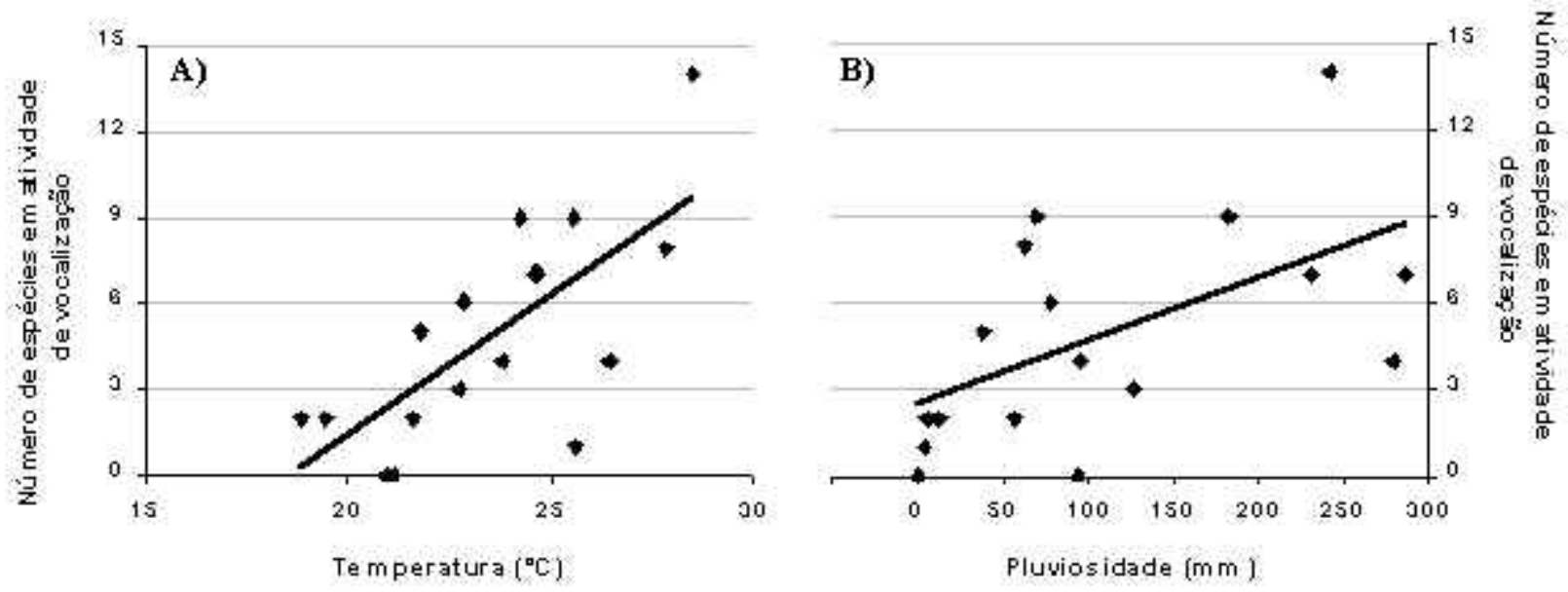

Figura 3. Regressão linear entre os fatores ambientais: temperatura média mensal (A) e pluviosidade total mensal (B) vs. número de espécies encontradas em atividade de vocalização na Floresta Estadual "Edmundo Navarro de Andrade", Rio Claro, São Paulo. Cada ponto representa um mês de estudo. 